\title{
SEISMOLOGY OF THE ZZ CETI STARS
}

\author{
J. CHRISTOPHER CLEMENS \\ Palomar Observatory \\ 105-24, California Institute of Technology, Pasadena, CA 91125
}

\begin{abstract}
The pulsations of white dwarf stars are potentially a rich source of information about white dwarf structural properties. Extracting and applying this information to improve our knowledge of white dwarf interiors requires measuring individual eigenperiods in a complex power spectrum, and identifying the character of the eigenmodes they represent. This review will summarize observational progress in these areas for the ZZ Ceti pulsators.
\end{abstract}

\section{Introduction}

The ZZ Ceti stars are the coolest and most numerous of the three known classes of pulsating white dwarf stars. They are apparently normal DA (H atmosphere) white dwarfs, with effective temperatures appropriate for the formation of a $\mathrm{H}$ partial ionization zone near their surfaces $(12,500 \mathrm{~K})$. They exhibit non-radial $g$-mode pulsations with periods ranging from about $100-1000 \mathrm{~s}$, and amplitudes of $1-80 \mathrm{mmag}$. The dominant period exhibited by any individual star is anti-correlated with its temperature, i.e., cooler stars pulsate with longer periods (Winget and Fontaine, 1982; Clemens, 1993; Bergeron et al., 1995). The cooler stars also show larger amplitudes and more complex period spectra. The longer periods presumably result from the deeper convection zone, and the correspondingly longer thermal timescale at the region of strongest pulsational driving, although other factors may contribute (Brickhill, $1983 ; \mathrm{Wu}, 1997)$. The amplitude and complexity trends are not as well-explored theoretically, nor as well-quantified observationally.

The $g$-mode spectrum of theoretical models is suitably rich to accommodate the observed periods for many different choices of degree $l$ and radial order $n$. Thus it is difficult to interpret the measured periods (presumed to be eigenperiods) without some independent means of identifying the character of the associated eigenmodes. A variety of methods have been applied to the problem of mode identification. I will discuss some of them in this review, including a promising new effort using Keck II and the Low Resolution Imaging Spectrograph (LRIS; Oke et al., 1995). Before this discussion, I will draw attention to some recent observational results by Kanaan (1996) regarding the boundaries of the instability strip. 


\section{Driving Mechanisms and the Red Edge}

To drive pulsations, heat flowing from the highly conductive white dwarf interior through the radiative and convective zones near the surface must be converted to mechanical work. Most of the published theory has explored the $\kappa$ mechanism as the valve which modulates heat flow to produce pulsational driving (Dolez and Vauclair, 1981; Winget et al., 1982; Bradley and Winget, 1994). However, Brickhill (1991a), and more recently Wu (1997) have criticized this approach on the grounds that the models assume that the convective flux is not perturbed by the pulsations. Because convection can adjust on a timescale much shorter than the observed pulsation periods, Brickhill (1991) and Wu (1997) argue for the opposite assumption-that convection adjusts instantaneously to the pulsations. Under this assumption, they find pulsational driving via a mechanism Brickhill has named "convective driving". In convective driving, the convection zone itself drives pulsations by storing energy during compression, and releasing it upon expansion.

This is not a theoretical review, so I will not attempt to assess the merits of these models. The ultimate crucible for refining theory lies in observation, and Kanaan (1996) has recently presented observations which are relevant to the issue of pulsational driving and damping. One of the challenges for non-adiabatic pulsation theory is to match the location of the observed edges of the instability strip. Unfortunately (or fortunately, depending on your point of view) the temperature of the blue edge is sensitive to the convective efficiency assumed by the models (Bradley and Winget, 1994). So the precise location may be a good indicator of the appropriate convective efficiency to use in a model, but it is probably not a good way to choose between competing models for the pulsation driving mechanism.

The red edge offers more promise, because the models exhibit very different behavior there. Models relying on $\kappa$ driving continue pulsating as they cool to quite low temperatures or until some independent mechanism, such as violation of the surface boundary condition (Hansen, Winget and Kawaler, 1985), causes them to cease. Conversely, in the models for convective driving, the convection zone which drives the pulsations soon absorbs all of the flux modulation, and renders the pulsations undetectable at 11,500 K, at least in flux measurements (Brickhill, 1991b; Wu, 1997).

Observationally, the location of the red edge has been difficult to fix precisely, because searches for pulsation via conventional techniques were afflicted by atmospheric variations at low frequencies. Kanaan (1996) has addressed this problem through the use of high-speed CCD photometry, with interesting results. Figure 1 shows Kanaan's measured pulsation limits for stars cooler than the ZZ Cetis, along with the power measured in the known pulsators. The plot shows the gentle onset of pulsations at about $13,500 \mathrm{~K}$, a rise in amplitude at lower temperatures, and then a dramatic cutoff near 11,500 degrees. Below that temperature, no stars are found to pulsate with amplitudes greater than $4 \mathrm{mmag}$. Some care must be used in interpreting this diagram, since the known large amplitude pulsators vary in the total amount of power they display, and the plot shows the maximum observed for each star. Nonetheless, figure 1 indicates that the red edge is very abrupt, and offers a challenge to theoretical models, which must account both for the existence of pulsations and for their absence at the appropriate temperatures. 


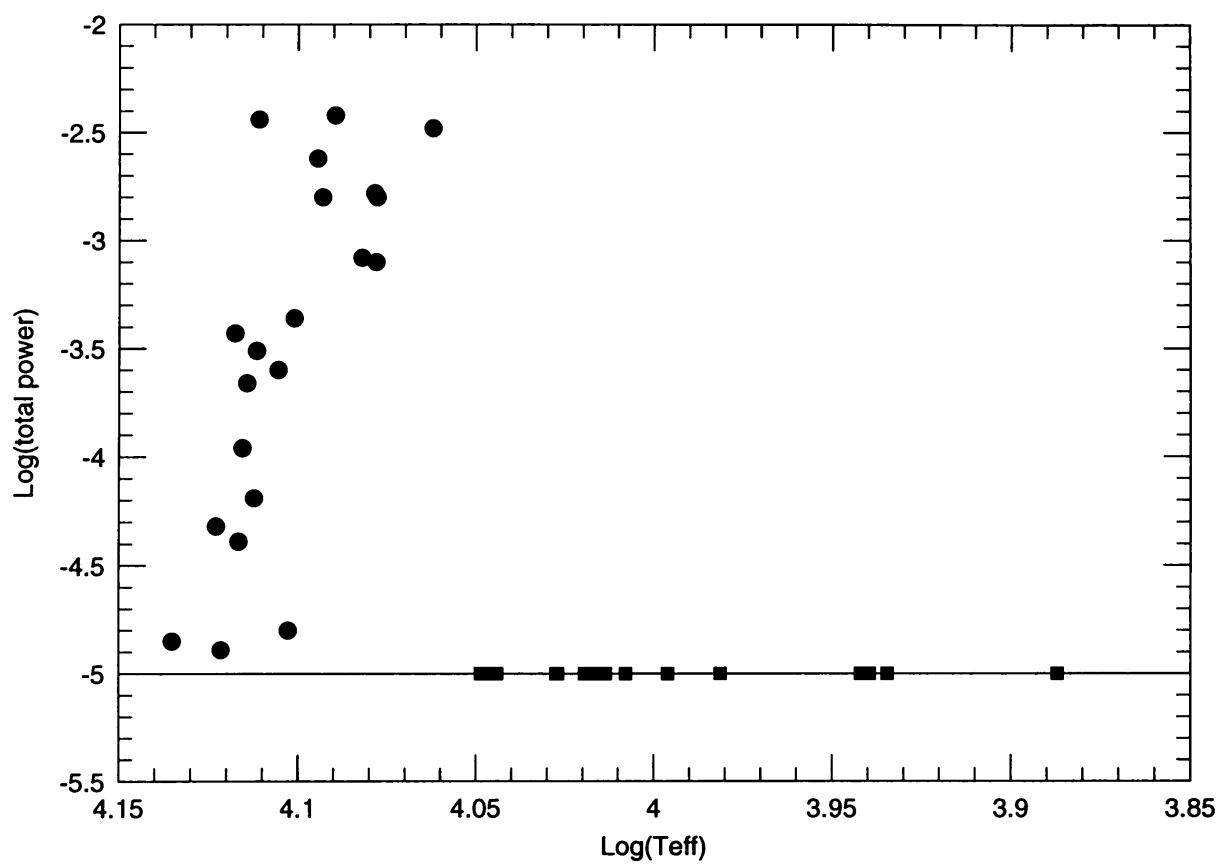

Figure 1. The pulsation power exhibited by ZZ Ceti stars and cooler DAs. Reprinted from Kanaan (1996)

\section{Asteroseismology}

Fortunately, while helpful, it is not absolutely necessary to know how pulsations are excited to use them for asteroseismological purposes. It is enough to have reliable structural models, whose eigenperiods can be calibrated against the measured periods. Currently, the goals of ZZ Ceti asteroseismology are to measure stellar masses $\left(M_{*}\right)$ and, particularly, the masses of the surface $\mathrm{H}$ layers $\left(M_{H}\right)$. Measuring $M_{H}$ for a significant sample of $\mathrm{ZZ}$ Cetis can help resolve an ongoing debate concerning the nature of DA white dwarfs, and the source of the division between DA and non-DA spectral types. Shipman (1997) and Fontaine and Wesemael (1997) give recent reviews of this topic.

Measuring $M_{H}$ is possible in principle because of the abrupt change in mean molecular weight, and therefore density, at the interface between the surface $\mathrm{H}$ layer and the underlying layer (presumed to be $\mathrm{He}$ ). This interface perturbs the eigenperiods from the value they would have in a homogeneous star (mode trapping; see Winget, 1981). The size and nature of these changes are a diagnostic of $M_{H}$. Actually measuring $M_{H}$ requires comparing the measured mode periods for a star to modes of corresponding $n$ and $l$ in the models. Identifying the $n$ and $l$ of observed modes has been the main challenge, and the main obstacle, to ZZ Ceti seismology.

Clemens (1993) has suggested a scheme for answering limited questions about $M_{H}$, without the need for mode identification. In the models for the production of white dwarfs from Asymptotic Giant Branch (AGB) stars, the DAs are those stars 

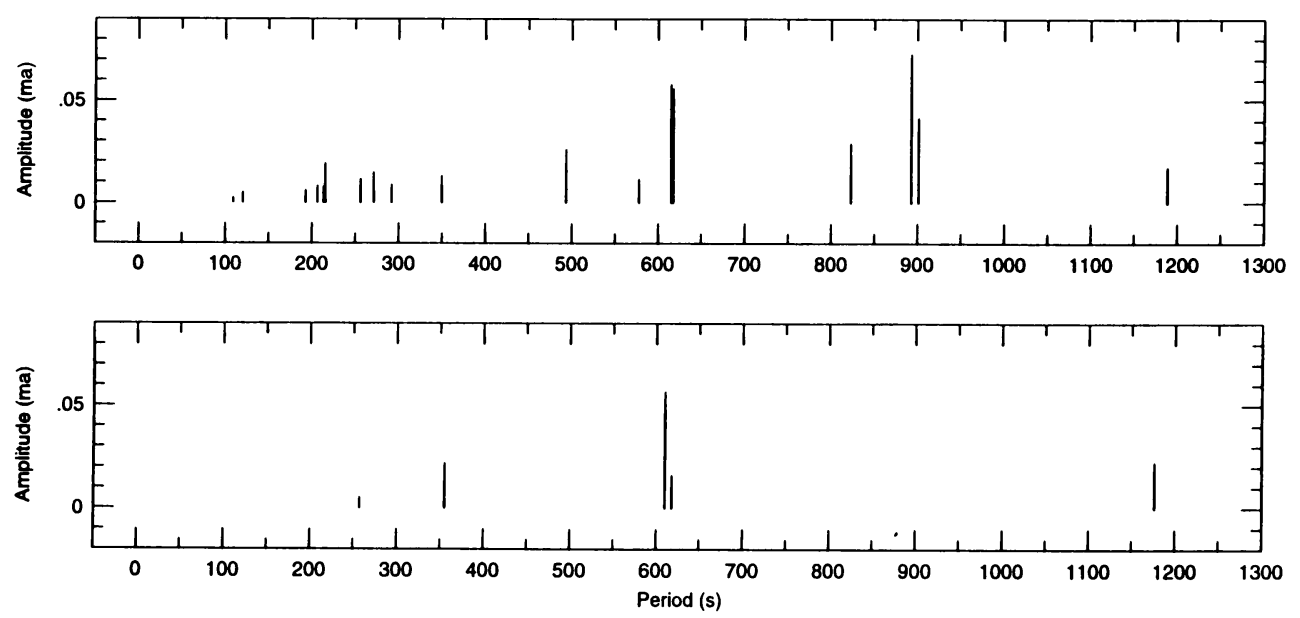

Figure 2. Comparison of the largest modes of ZZ Ceti stars compiled in 1993 (upper panel) with the largest modes of newly discovered ZZ Cetis (lower panel).

that survive their He shell flashes with enough surface $H$ to sustain residual burning until well after they depart the AGB (Iben 1984). We know that DA white dwarfs have a narrow distribution in total mass (Bergeron, Saffer and Liebert 1992), and the Iben models further predict that $M_{H}$, ultimately fixed by the physics of nuclear burning, will also be narrowly distributed near $10^{-4} M_{\odot}$. Since $M_{*}$ and $M_{H}$ are the dominant quantities affecting mode periods (Brassard et al., 1992; Bradley, 1996), this theory suggests eigenmodes of a specific $n$ and $l$ in different stars will be narrowly distributed about a single period. Conversely, the competing model composed by Fontaine and Wesemael (1987) to explain observed changes in the ratio of DA to non-DA stars requires a large range of $M_{H}$, and a correspondingly wide distribution of periods for the same mode in different stars.

By intercomparing the measured periods of all the ZZ Ceti's, Clemens (1993) found evidence for period groupings, supporting the models of Iben (1984). Clemens suggested that ZZ Cetis discovered in the future could be used to test the reality of these groupings. There have been five ZZ Cetis discovered since that time (Stobie et al., 1995; Giovannini et al., 1997, Vauclair et al., 1997; Jordan et al., 1997). Figure 2 shows the largest modes in each of these stars along with the largest modes of those collected by Clemens in 1993. There remains a clear tendency of modes to group around certain periods. The group near 615 seconds is particularly striking. It is difficult to avoid the conclusion that these stars share a common physical structure, as predicted by the evolutionary models of Iben (1984).

\section{Mode Identification}

Although figure 2 represents an ensemble argument for similar structure among the ZZ Cetis, it cannot substitute, in the case of any single star, for independent mode identification. Using various schemes, mode identifications have been claimed for a 
total of five ZZ Ceti stars. In every case but one, the identifications are incomplete, and must be bolstered by additional assumptions to conduct seismological analysis. These analyses typically yield a set of constraints on $M_{H}$ and $M_{*}$.

Two independent groups have identified the $215 \mathrm{~s}$ mode in G117-B15A as $l=1$. Robinson et al. (1995) exploited the differing effects of limb darkening on mode amplitudes at ultraviolet wavelengths. All modes show larger amplitudes in the ultraviolet, where stronger limb darkening diminishes the effects of geometrical cancelation, but modes of $l=2$ rise faster in amplitude than those of $l=1$. Robinson et al. found ultraviolet amplitudes consistent with $l=1$ for the $215 \mathrm{~s}$ mode in G117-B15A. Fontaine et al. (1996) measured $l$ for the same star by assuming that the non-linearities in the light curve result from the transfer of linear temperature variations into non-linear flux variations. They found $l=1$ for all modes. These identifications demand that $M_{H}$ be greater than $1 \times 10^{-6} M_{\odot}$. Without assuming some value for $n$ it is impossible to be more specific.

G226-29 represents the best example of an asteroseismological measurement of $M_{H}$. The only periods detected in this star form a uniformly spaced triplet at 109.3 s. Fontaine et al. (1992) assumed that this was an $l=1$ mode rotationally split into 3 components. They found that only for $M_{H}$ of about $10^{-4} M_{\odot}$ could any $l=1$ mode be so short. Kepler et al. (1997) have recently completed the analysis of ultraviolet data from the Hubble Space Telescope and verified that at least two of the triplet members are $l=1$, so this is now a real measurement of $M_{H}$ rather than a constraint.

The remaining three stars are GD165, G29-38 and GD154. These have tentative mode identifications based on multiplet splittings, period spacings and trapped-mode spacings, respectively. The values of $M_{H}$ in units of solar mass are $M_{H}>10^{7}$ for GD165 (Bergeron et al. 1993), $M_{H}>4 \times 10^{-7}$ for G29-38 (Bradley and Kleinman, 1997), and $M_{H} \approx 10^{-10}$ for GD154 (Pfeiffer et al. , 1996).

In summary, four out of five of the published identifications give $M_{H}$ consistent with $\sim 10^{-4} M_{\odot}$; the fifth does not. The best measurement, that for G226-29, gives exactly the value predicted by models of Iben (1984), and preferred by the ensemble analysis presented by Clemens (1993). Consequently, the results of the few detailed asteroseismological analyses that currently exist are entirely consistent with results Clemens (1993) obtained by intercomparing ZZ Ceti period spectra.

\section{The Future}

Finally, one more interesting development was presented in the poster session of this symposium by van Kerkwijk et al. Time resolved spectroscopy using LRIS on the Keck II telescope has revealed the radial velocities associated with pulsations in the large amplitude ZZ Ceti star G29-38. Figure 3 shows a time series of the velocity shifts measured from Balmer lines in the spectrum. The lower panel shows the corresponding Fourier transform. Undoubtedly, the measured velocities originate from horizontal motions viewed at the stellar limb; the amplitude of the radial motions in the white dwarf's extreme gravity is immeasurably small. The periods of the radial velocity variations are the same as those measured in flux, with the following notable exceptions: the "combination" peaks appearing at sums and differences of the main power in the flux variations are not present in the velocity measurements.

The detection and measurment of pulsation velocities in ZZ Ceti stars will have numerous consequences we are only beginning to explore. First, they allow a more direct measurement of eigenmode amplitudes, one not afflicted by the non-linearities 

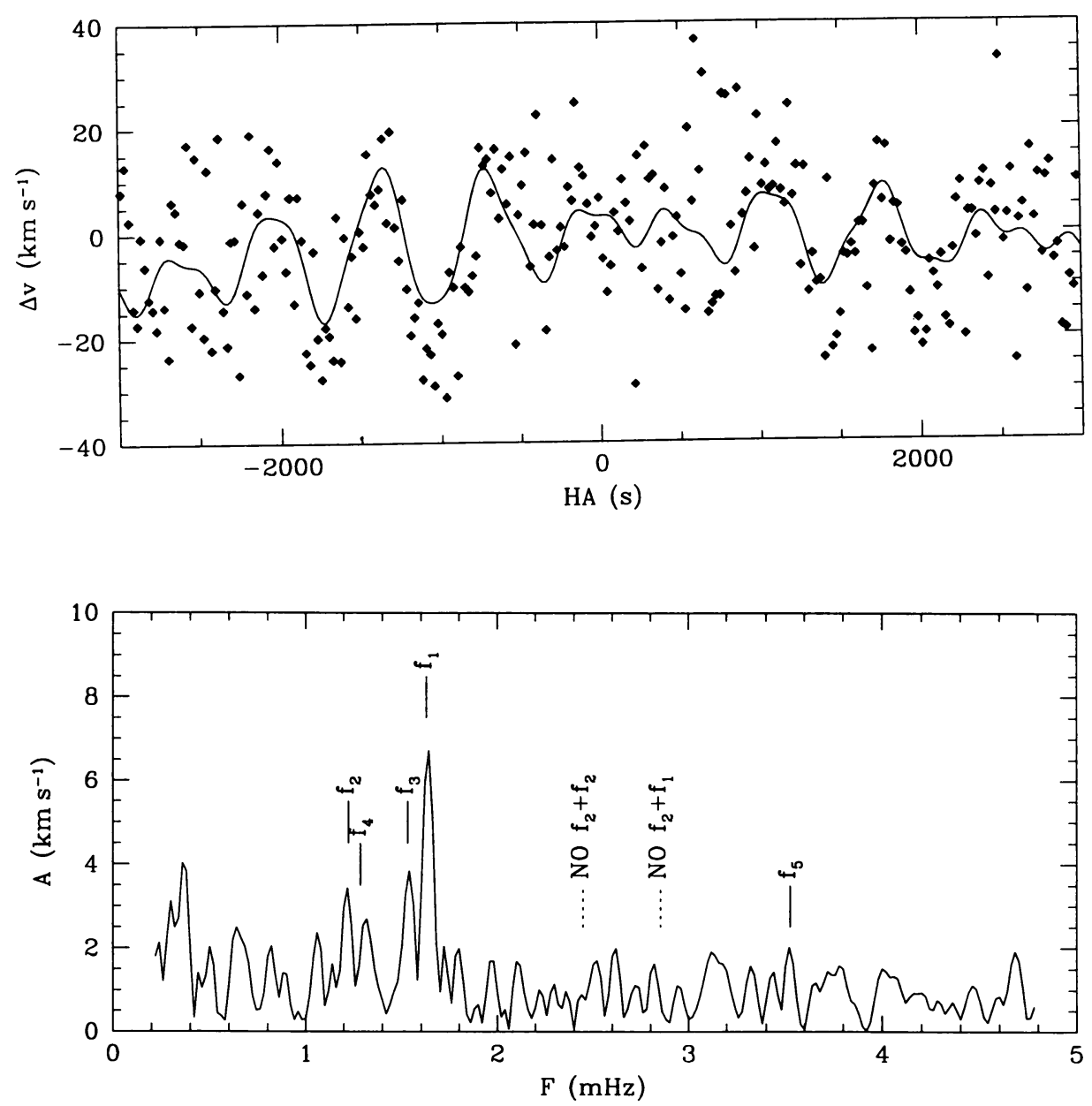

Figure 3. Radial velocity curve for the star G29-38 (upper panel), and its Fourier transform (lower panel). The frequency labels $f_{1}$ through $f_{5}$ correspond to the five largest frequencies found in the flux modulations. The sum frequencies $f_{2}+f_{2}$ and $f_{2}+f_{1}$ do not appear here, although they are larger than $f_{5}$ in the Fourier transform of the flux variations.

displayed in flux. This will allow a more direct comparison to the amplitudes calculated from non-adiabatic pulsation models. Furthermore, they offer, for the first time, a way to select normal modes without the confusion arising from combination frequencies. Finally, they offer a new method of mode identification, because the ratio of velocity to light amplitude varies with $l$. Continued observations of more ZZ Ceti stars with the Keck telescope promises to provide an entirely new dimension to the study of ZZ Ceti pulsations. 


\section{References}

Bergeron, P., Fontaine, G., Brassard, P., Lamontagne, R., Wesemael, F., Winget, D. E., Nather, R. E., Bradley, P. A., Claver, C. F., Clemens, J. C., Kleinman, S. J., Provencal, J., McGraw, J. T., Birch, P., Candy, M., Buckley, D. A., Tripe, P., Augusteijn, T., Vauclair, G., Kepler, S. O., and Kanaan, A.: 1993, Astron. J. 106, 1987.

Bergeron, P., Saffer, R. A., and Liebert, J.: 1992, Astrophys. J. 394, 228.

Bergeron, P., Wesemael, F., Lamontagne, R., Fontaine, G., Saffer, R. A., and Allard, N. F.: 1995, Astrophys. J. 449, 258.

Bradley, P. A.: 1996, Astrophys. J. 468, 350.

Bradley, P. and Kleinman, S.: 1997, in J. Isern, M. Hernanz, and E. Garcia-Berro (eds.), White Dwarfs, Kluwer Academic Publishers, Dordrecht, p. 445.

Bradley, P. A., and Winget, D. E.: 1994, Astrophys. J. 421, 236.

Brassard, P., Fontaine, G., Wesemael, F., and Tassoul, M.: 1992, Astrophys. J. Suppl. 81, 747.

Brickhill, J. A.: 1983, Monthly Notices Roy. Astron. Soc. 204, 537.

Brickhill, J. A.: 1991a, Monthly Notices Roy. Astron. Soc. 251, 673.

Brickhill, J. A.: 1991b, Monthly Notices Roy. Astron. Soc. 252, 334.

Clemens, J. C.: 1993, Baltic Astronomy 2, 407.

Dolez, N., and Vauclair, G.: 1981, Astron. Astrophys. 102, 375.

Fontaine, G., Brassard, P., Bergeron, P., and Wesemael, F.: 1996, Astrophys. J. 399, L91.

Fontaine, G., Brassard, P., Bergeron, P., and Wesemael, F.: 1996, Astrophys. J. 469, 320.

Fontaine, G., and Wesemael, F.: 1987, in A. G. D. Philip, D. S. Hayes, and J. Liebert (eds.), The Second Conference on Faint Blue Stars, IAU Colloq. 95, L. Davis Press, Schenectady, p. 319.

Fontaine, G., and Wesemael, F.: 1997, in J. Isern, M. Hernanz, and E. Garcia-Berro (eds.), White Dwarfs, Kluwer Academic Publishers, Dordrecht, p. 165.

Giovannini, O., Kepler, S. O., Kanaan, A., Costa, A. F. M., and Koester, D.: 1997, Astron. Astrophys. in press.

Hansen, C. J., Winget, D. E., and Kawaler, S. D.: 1985, Astrophys. J. 295, 547.

Iben, I., Jr.: 1984, Astrophys. J., 277, 333.

Jordan, S., Koester, D., Vauclair, G., Dolez, N., Heber, U., Hagen, H.-J., Reimers, D., Chevreton, M., and Dreizler, S.: 1997, Astron. Astrophys. in press.

Kanaan, A.: 1996, PhD Thesis, University of Texas.

Kepler, S. O., et al.: 1997, Baltic Astronomy in press.

Oke, J. B., Cohen, J. G., Carr, M., Cromer, J., Dingizian, A., Harris, F. H., Labrecque, S., Lucinio, R., Schaal, W., Epps, H., and Miller, J.: 1995, Publ. Astron. Soc. Pacific 107, 375.

Pfeiffer, B., Vauclair, G., Dolez, N., Chevreton, M., Fremy, J.-R., Barstow, M., Belmonte, J. A., Kepler, S. O., Kanaan, A., Giovannini, O., Fontaine, G., Bergeron, P., Wesemael, F., Grauer, A. D., Nather, R. E., Winget, D. E., Provencal, J., Clemens, J. C., Bradley, P. A., Dixson, J., Kleinman, S. J., Watson, T. K., Claver, C. F., Matzeh, T., Leibowitz, E. M., and Moskalik, P.: 1996, Astron. Astrophys. 314, 182.

Robinson, E. L., Mailloux, T. M., Zhang, E., Koester, D., Stiening, R. F., Bless, R. C., Percival, J. W., Taylor, M. J., and Van Citters, G. W.: 1995, Astrophys. J. 438, 908.

Shipman, H. L.: 1997, in J. Isern, M. Hernanz, and E. Garcia-Berro (eds.), White Dwarfs, Kluwer Academic Publishers, Dordrecht, p. 165.

Stobie, R. S., O'Donoghue, D., Ashley, R., Koen, C., Chen, A., and Kilkenny, D.: 1995 Monthly Notices Roy. Astron. Soc. 272, L21.

Vauclair, G., Dolez, N., Jian Ning, F., and Chevreton, M.: 1997, Astron. Astrophys. 322, 155.

Winget, D. E.: 1981, PhD Thesis, University of Rochester.

Winget, D. E. and Fontaine, G.: 1982, in J. P. Cox and C. J. Hansen (eds.), Pulsations in Classical and Cataclysmic Variable Stars, University of Colorado Press, Boulder, p. 46.

Winget, D. E., Van Horn, H. M., Tassoul, M., Hansen, C. J., Fontaine, G., and Carrol, B. 
W.: 1982, Astrophys. J. 253, L29.

Wu, Y.: 1997, in J. Isern, M. Hernanz, and E. Garcia-Berro (eds.), White Dwarfs, Kluwer Academic Publishers, Dordrecht, p. 467. 